\title{
Leishmania infantum heat shock protein 83 for the serodiagnosis of tegumentary leishmaniasis
}

\footnotetext{
B.J. Celeste ${ }^{1}$,

S.O. Angel ${ }^{5}$,

L.G.M. Castro²,

M. Gidlund ${ }^{4}$ and $\mathrm{H}$. Goto ${ }^{1,3}$
}

\author{
${ }^{1}$ Laboratório de Soroepidemiologia e Imunobiologia, \\ Instituto de Medicina Tropical de São Paulo, Departamentos de \\ ${ }^{2}$ Dermatologia and ${ }^{3}$ Medicina Preventiva, Faculdade de Medicina, and \\ ${ }^{4}$ Laboratório de Imunofisiologia, Departamento de Imunologia, \\ Instituto de Ciências Biomédicas, Universidade de São Paulo, São Paulo, SP, Brasil \\ ${ }^{5}$ Departamento de Parasitologia, ANLIS Dr. Carlos G. Malbran, \\ Buenos Aires, Argentina
}

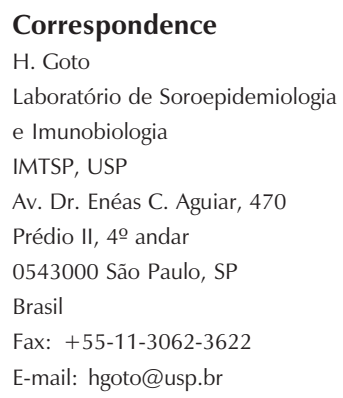

\begin{abstract}
The serologic assay is an important tool in the diagnosis of leishmaniasis. One of the most commonly used tests is enzyme-linked immunosorbent assay (ELISA). Since total Leishmania promastigotes are used as antigen in the routine assay, false-positive reactions are frequent due to cross-reaction with sera from other diseases, mainly Chagas' disease. Therefore, an antigen that determines less cross-reactivity has been pursued for the serodiagnosis of leishmaniasis. In the present study we analyzed the use of recombinant Leishmania infantum heat shock protein (Hsp) 83 in ELISA for the serodiagnosis of cutaneous $(\mathrm{N}=12)$ and mucocutaneous leishmaniasis $(\mathrm{N}=14)$ and we observed the presence of anti-L. infantum Hsp 83 antibodies in all samples as well as anti-Leishmania total antigen antibodies. When cross-reactivity was tested, chronic Chagas' disease patients $(\mathrm{N}=10)$ did not show any reactivity. Therefore, we consider this $L$. infantum Hsp 83 to be a good antigen for routine use for serodiagnosis of tegumentary leishmaniasis.
\end{abstract}

Key words

- Leishmania infantum

- Hsp 83

- ELISA

- Serodiagnosis

- Tegumentary leishmaniasis

- Chagas' disease
Leishmaniasis is a disease caused by protozoa of the genus Leishmania which can occur in the cutaneous, mucocutaneous or visceral form. Serological tests are most commonly used for diagnosis and, in addition to immunofluorescence, enzyme-linked immunosorbent assay (ELISA) is the assay of choice because of its sensitivity and higher predictive value (1). However, since the antigen used for anti-Leishmania ELISA is a total promastigote antigen, false-positive reactions are frequent when sera from patients with other diseases, mainly Chagas' disease, are tested (2-4). Therefore, an antigen with less cross-reactivity has been pursued for the serodiagnosis of leishmaniasis.

Several investigators have reported heat shock proteins (Hsp) from Leishmania belonging to the $60,70,83$, and 90 families that have been tested for the serodiagnosis of leishmaniasis. L. major Hsp 60 were tested with cutaneous leishmaniasis sera (5), $L$. braziliensis Hsp 83 and Hsp 70 with cutaneous, mucocutaneous and diffuse cutaneous leishmaniasis sera (6), and $L$. donovani $\mathrm{Hsp}$ 70 (7) and Hsp 83 of L. infantum with canine 
visceral leishmaniasis sera (8). Except for $L$. donovani Hsp 70, all sera reacted with samples from different leishmaniasis patients. In the study with L. infantum Hsp 70, how-

Table 1. Titers and median of anti-Hsp 83 and anti-Leishmania major-like total antigen (anti-leishmania) in mucocutaneous and cutaneous leishmaniasis patients.

\begin{tabular}{|c|c|c|c|c|c|}
\hline \multicolumn{3}{|c|}{ Mucocutaneous leishmaniasis } & \multicolumn{3}{|c|}{ Cutaneous leishmaniasis } \\
\hline Patient & anti-Hsp 83 & anti-leishmania & Patient & anti-Hsp 83 & anti-leishmania \\
\hline $\mathrm{GC}$ & 320 & 5120 & GCS & 80 & 40 \\
\hline MLC & 640 & 20240 & GNS & 320 & 2560 \\
\hline JM & 320 & 640 & EMR & 320 & 320 \\
\hline YM & 160 & 160 & DMR & 160 & 160 \\
\hline BEA & 160 & 320 & CJS & 640 & 320 \\
\hline ACS & 1280 & 1280 & NSA & 640 & 160 \\
\hline $\mathrm{OL}$ & 320 & 320 & JVS & 320 & 40 \\
\hline AMM & 160 & 640 & CAA & 160 & 1280 \\
\hline MNP & 640 & 640 & RDO & 320 & 640 \\
\hline JFS & 80 & 640 & MDM & 80 & 1280 \\
\hline $\mathrm{FR}$ & 1280 & 640 & AMP & 80 & 160 \\
\hline AMS & 160 & 320 & ESC & 320 & 640 \\
\hline EVL & 160 & 160 & & & \\
\hline AFS & 320 & 5120 & & & \\
\hline Median & 320 & 640 & Median & 320 & 320 \\
\hline
\end{tabular}

ELISA-IgG test. Positive when titers were $\geq 40$.

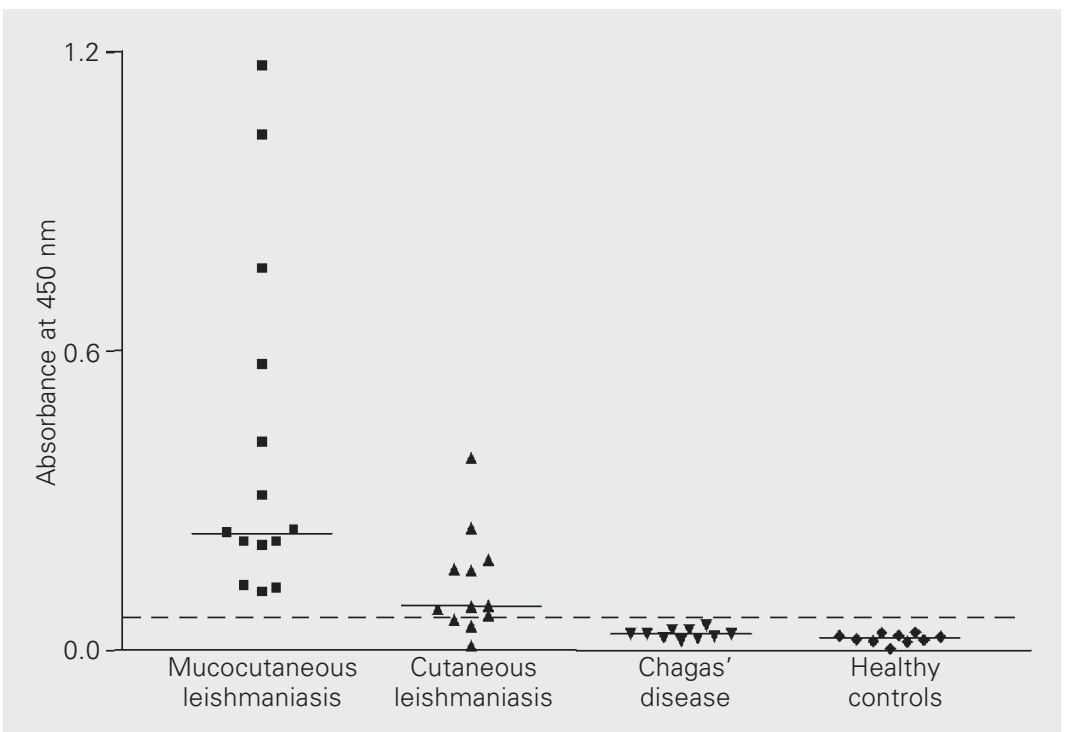

Figure 1. Reactivity of sera from cutaneous $(N=12)$ and mucocutaneous ( $N=14$ ) leishmaniasis patients, Chagas' disease $(N=10)$ patients and healthy controls $(N=10)$ with recombinant $L$. infantum (Hsp 83) in the ELISA-lgG test. The broken line indicates the cutoff values, corresponding to the mean absorbance value of the control sera $+2 \mathrm{SD}$. The solid horizontal lines indicate the median value. ever, in addition to the expected reactivity with visceral and mucocutaneous leishmaniasis sera, cross-reactivity was observed with sera from Chagas' disease patients (9).

In the present study we analyzed the recombinant L. infantum Hsp 83 (8) using ELISA for serodiagnosis of tegumentary leishmaniasis, we compared the performance with the L. major-like total antigen used in routine tests (9) and we analyzed the cross reactivity with Chagas' disease samples. The recombinant protein was produced and purified by Ni-NTA affinity chromatography (QIAGEN GmbH, Hilden, Germany) according to the method of Angel et al. (8) and the anti-Hsp 83 antibody was detected by ELISA. Briefly, microplates were coated with $50 \mu \mathrm{l} /$ well of recombinant antigen $(1 \mathrm{mg} / \mathrm{ml} L$. infantum Hsp 83) diluted in $60 \mathrm{mM}$ sodium carbonate buffer, $\mathrm{pH}$ 9.6. The sera and peroxidase-conjugate anti-human IgG antibody were diluted in PBS-T $(0.15 \mathrm{M} \mathrm{NaCl}, 10 \mathrm{mM}$ sodium phosphate, $\mathrm{pH} 7.2,0.05 \%$ Tween20) containing $1 \%$ bovine serum albumin (Sigma, St. Louis, MO, USA). The substrate used was $5.2 \mathrm{mM} 5$-aminosalicylic acid, and $1.5 \mathrm{mM} \mathrm{H}_{2} \mathrm{O}_{2}$. The reaction was stopped by the addition of $25 \mu \mathrm{l}$ of $1 \mathrm{M} \mathrm{NaOH}$ to each well and absorbance was measured at 450 $\mathrm{nm}$. Cut-off of the test corresponded to mean plus 2 standard deviations.

We analyzed sera from patients with cutaneous $(\mathrm{N}=12)$ and mucocutaneous $(\mathrm{N}=$ 14) leishmaniasis examined in the Department of Dermatology, University of São Paulo Medical School. All sera had been sent for routine serological tests for leishmaniasis. The diagnosis was confirmed in all cases by a positive Montenegro skin test (10), induration $\geq 5 \mathrm{~mm}$, and/or by histopathology of the skin lesion (11), and by the detection of anti-Leishmania antibody by the immunofluorescence test. Sera from 10 normal blood bank donors were used as controls and sera from 10 Chagas' disease patients (kindly provided by M.G. Valin from the Immunology Section of the Laboratório 
Central, HC-FMUSP) were also analyzed. The research protocol was analyzed and approved by the Ethics Committees of the Instituto de Medicina Tropical de São Paulo, USP, and of the Heart Institute, Hospital das Clínicas, Faculdade de Medicina, USP.

All patients with tegumentary leishmaniasis showed the presence of anti-L. infantum Hsp 83 antibodies (Table 1). When we compared the reactivity to Hsp 83 and Leishmania total antigen we found some variations but all samples were positive for both antigens (Table 1). When cross-reactivity was tested with Hsp 83 antigen, sera from Chagas' disease patients did not show any reactivity (Figure 1). When the same serum samples were tested with Leishmania total antigen they all showed cross-reactivity (median absorbance $=0.608$, range: $0.165-0.737$, cutoff $=0.158$ ).

The absence of cross-reactivity with Chagas' disease sera is important since the endemic areas for both diseases often overlap. The carboxyl-terminal region of $L$. (Viannia) braziliensis Hsp 70 showed no cross-reactivity with Chagas' patient sera, but this antigen did not react with sera from cutaneous leishmaniasis patients infected with $L$. amazonensis (12). Thus, this antigen is not suitable for use in diagnosis in Brazil, where patients from some regions are infected with these species of Leishmania. The L. infantum Hsp 70-derived 50-mer peptide also showed no cross-reactivity with sera from Chagas' disease patients (9). However, compared with the recombinant $L$. infantum Hsp 83 used in the present study, the synthesis of a peptide is technically more complex than the production of a recombinant antigen. Therefore, we consider this $L$. infantum Hsp 83 to be a good antigen for routine use for serodiagnosis of tegumentary leishmaniasis.

\section{References}

1. Guimarães MCS, Celeste BJ, Franco EL, Cucé LC \& Belda Jr W (1989). Evaluation of serological diagnostic indices for mucocutaneous leishmaniasis, immunofluorescence tests and enzyme-linked immunoassay, for $\operatorname{lgG}, \lg \mathrm{M}$ and $\lg \mathrm{A}$ antibodies. Bulletin of the World Health Organization, 67: 643-648.

2. Roffi J, Dedet JP, Desjeux P \& Garré MT (1980). Detection of circulating antibodies in cutaneous leishmaniasis by enzyme-linked immunosorbent assay (ELISA). American Journal of Tropical Medicine and Hygiene, 29: 183-189.

3. Guimarães MCS, Celeste BJ, Corrales LEM \& Antunes CMF (1991). Comparison on the performance of Leishmania major-like and Leishmania braziliensis braziliensis as antigen for new world leishmaniasis lgG-immunofluorescence test. Revista do Instituto de Medicina Tropical de São Paulo, 33: 503-508.

4. Kalter DC (1994). Laboratory tests for the diagnosis and evaluation of leishmaniasis. Dermatologic Clinics, 12: 37-51.

5. Rey-Ladino JA, Joshi PB, Singh B, Gupta R \& Reiner NE (1997). Leishmania major-like: molecular cloning, sequencing, and expression of the heat shock protein 60 gene reveals unique carboxy terminal peptide sequences. Experimental Parasitology, 85: 249263.

6. Skeiky YAW, Benson DR, Costa JLM, Badaró R \& Reed SG (1997). Association of Leishmania heat-shock protein 83 antigen and immunoglobulin G4 antibody titers in Brazilian patients with diffuse cuta- neous leishmaniasis. Infection and Immunity, 65: 5368-5370.

7. MacFarlene J, Blaxter ML, Bishop RP, Miles MA \& Kelly JM (1990). Identification and characterisation of a Leishmania donovani antigen belonging to the $70-\mathrm{kDa}$ heat-shock protein family. European Journal of Biochemistry, 190: 377-384.

8. Angel SO, Requena JM, Soto M, Criado D \& Alonso C (1996). During canine leishmaniasis a protein belonging to the 83-kDa heat-shock protein family elicits a strong humoral response. Acta Tropica, 62: 45-56.

9. Quijada L, Requena JM, Soto M \& Alonso C (1998). Analysis of the antigenic properties of the L. infantum Hsp 70: design of the synthetic peptides for specific serodiagnosis of human leishmanasis. Immunology Letters, 63: 169-174.

10. Montenegro J (1926). A cutis-reação na Leishmaniose. Anais da Faculdade de Medicina da Universidade de São Paulo, 1: 323-330.

11. Cuba Cuba CA, Marsden PD, Barreto AC, Rocha R, Sampaio RR \& Patzlaff $L$ (1981). Parasitologic and immunologic diagnosis of American (mucocutaneous) leishmaniasis. Bulletin of the Pan American Health Organization, 15: 249-259.

12. Amorim AG, Carrington M, Miles MA, Barker DC \& de Almeida ML (1996). Identification of C-terminal region of $70 \mathrm{kDa}$ heat shock protein from Leishmania (Viannia) braziliensis as a target for the humoral immune response. Cell Stress Chaperones, 1: 177-187. 